\title{
FIXED POINTS AND CYCLIC CONTRACTION MAPPINGS UNDER IMPLICIT RELATIONS AND APPLICATIONS TO INTEGRAL EQUATIONS
}

\author{
HEMANT KUMAR NASHINE
}

\begin{abstract}
Inspired by the fact that the discontinuous mappings cannot be (Banach type) contractions and cyclic contractions need not be continuous, and taking into account that there are applications to integral and differential equations based on cyclic contractions, a new type of cyclic contraction mappings satisfying an implicit relation that involves a control function for a map in a metric space is originated. As a result, we derive existence and uniqueness results of fixed points for such mappings. We furnish suitable examples to demonstrate the validity of the hypotheses of our results. The results will be applied to the study of the existence and uniqueness of solutions for a class of nonlinear integral equations.
\end{abstract}

\section{INTRODUCTION AND PRELIMINARIES}

The Banach Contraction Principle is a very popular tool which is used to solve existence problems in many branches of Mathematical Analysis and its applications. It is no surprise that there is a great number of generalizations of this fundamental theorem. They go in several directions-modifying the basic contractive condition or changing the ambiental space. This celebrated theorem can be stated as follow.

Theorem 1.1. [1]. Let $(X, d)$ be a complete metric space and $T$ be a mapping of $X$ into itself satisfying:

$$
d(T x, T y) \leq k d(x, y), \forall x, y \in X,
$$

where $k$ is a constant in $(0,1)$. Then, $T$ has a unique fixed point $x^{*} \in X$.

Inequality (1) implies continuity of $T$. A natural question is whether we can find contractive conditions which will imply existence of a fixed point in a complete metric space but do not imply continuity.

2010 Mathematics Subject Classification. Primary: 54H25; Secondary: 47H10.

Key words and phrases. Fixed point, cyclic contraction, implicit relation, integral equation. 
There is in the literature a great number of generalizations of the Banach contraction principle (see [2] and references cited therein).

On the other hand, cyclic representations and cyclic contractions were introduced by Kirk et al. [3]. A mapping $T: A \cup B \rightarrow A \cup B$ is called cyclic if $T(A) \subseteq B$ and $T(B) \subseteq A$, where $A, B$ are nonempty subsets of a metric space $(X, d)$. Moreover, $T$ is called a cyclic contraction if there exists $k \in(0,1)$ such that $d(T x, T y) \leq k d(x, y)$ for all $x \in A$ and $y \in B$. Notice that although a contraction is continuous, cyclic contractions need not be. This is one of the important gains of this theorem.

Definition 1.1 (See $[3,4])$. Let $(X, d)$ be a metric space. Let $p$ be a positive integer, $A_{1}, A_{2}, \ldots, A_{p}$ be nonempty subsets of $X, Y=\cup_{i=1}^{p} A_{i}$ and $T: Y \rightarrow Y$. Then $Y$ is said to be a cyclic representation of $Y$ with respect to $T$ if

(i) $A_{i}, i=1,2, \ldots, p$ are nonempty closed sets, and

(ii) $T\left(A_{1}\right) \subseteq A_{2}, \ldots, T\left(A_{p-1}\right) \subseteq A_{p}, T\left(A_{p}\right) \subseteq A_{1}$.

Following in [3], a number of fixed point theorems on cyclic representations of $Y$ with respect to a self-mapping $T$ have appeared (see e.g. $[4,5,6,7,8,9,10,11])$.

In recent years, Popa [12] used implicit functions rather than contraction conditions to prove fixed point theorems in metric spaces whose strength lies in its unifying power as an implicit function can cover several contraction conditions at the same time which includes known as well as unknown contraction conditions. This fact is evident from examples furnished in Popa [12]. Implicit relations on metric spaces have been used in many articles (for detail see [13] - [20] and references cited therein).

In this paper, we introduce a new class of cyclic contraction mappings satisfying an implicit relation that involves a control function for a map in the setup of metric spaces, and then derive the existence and uniqueness of fixed points for such mappings. Suitable examples are provided to demonstrate the validity of our results. Our main result generalizes and improves many existing theorems in the literature. We also give an application of the presented theorems in the area of integral equations and prove an existence theorem for solutions of a system of integral equations.

\section{MAin Results}

In this section, first we define a suitable implicit function (given in [19]) involving six real non-negative arguments to prove our results.

Definition 2.1. [19]. Let $\digamma$ be the family of all real continuous functions $T: \mathbb{R}^{+^{6}} \rightarrow \mathbb{R}$, which are non-decreasing in the first variable and 
non-increasing in the third, fourth, fifth and sixth variables, satisfying the following conditions:

$\left(T_{1}\right)$ There exists $h \in[0,1)$ such that for all $u, v, w \geq 0$ with $T(u, v, v, u$, $w, 0) \leq 0$ or $T(u, v, u, v, 0, w) \leq 0$, we have $u \leq h v$;

$\left(T_{2}\right) T(u, u, 0,0, u, u)>0$ for every $u>0$.

It is easy to see that $T: \mathbb{R}^{+^{6}} \rightarrow \mathbb{R}$ belongs to $\digamma$ in each of the following cases:

Example 2.1. $T\left(t_{1}, \ldots, t_{6}\right)=t_{1}-c t_{2}-a\left(t_{3}+t_{4}\right)-b \sqrt{t_{5} t_{6}}$, where $c>0$, $a, b \geq 0$ and $c+\max \{2 a, b\}<1$;

Example 2.2. $T\left(t_{1}, \ldots, t_{6}\right)=t_{1}\left(t_{1}-a t_{2}-b t_{3}-c t_{4}\right)-d t_{5} t_{6}$, where $a>0$, $b, c, d \geq 0$ and $a+\max \{b+c, d\}<1$;

Example 2.3. $T\left(t_{1}, \ldots, t_{6}\right)=t_{1}-a t_{2}-b t_{3}-c t_{4}-d \min \left\{t_{5}, t_{6}\right\}$, where $a>0, b, c, d \geq 0$ and $a+\max \{b+c, d\}<1$;

Example 2.4. $T\left(t_{1}, \ldots, t_{6}\right)=t_{1}^{2}-a t_{2}^{2}-b \frac{1}{1+t_{3}+t_{4}} \min \left\{t_{5}^{2}, t_{6}^{2}\right\}$, where $a>0$, $b \geq 0$ and $a+b<1$;

Example 2.5. $T\left(t_{1}, \ldots, t_{6}\right)=t_{1}^{2}-a t_{2}^{2}+b \frac{t_{3} t_{4}}{1+t_{5}+t_{6}}$, where $0<a<1$ and $b \geq 0$.

To give the new notion of a cyclic contractive mapping, we need following concept:

We denote by $\Psi$ the set of functions $\psi:[0,+\infty) \rightarrow[0,+\infty)$ is a nondecreasing and continuous function satisfying:

$\left(\psi_{1}\right) \psi(t)<t$ for $t>0$ and $\psi(0)=0$;

$\left(\psi_{2}\right) \psi$ is subadditive, that is, for every $\mu_{1}, \mu_{2} \in[0,+\infty), \psi\left(\mu_{1}+\mu_{2}\right) \leq$ $\psi\left(\mu_{1}\right)+\psi\left(\mu_{2}\right)$

$\left(\psi_{3}\right)$ For all $t \in(0, \infty), \lim _{n \rightarrow \infty} t_{n}=0$ if and only if $\lim _{n \rightarrow \infty} \psi\left(t_{n}\right)=0$.

Next, we introduce a new notion of cyclic contraction mapping satisfying implicit relation that involves a control function.

Definition 2.2. Let $(X, d)$ be a metric space. Let $p$ be a positive integer, $A_{1}, A_{2}, \ldots, A_{p}$ be nonempty subsets of $X$ and $Y=\cup_{i=1}^{p} A_{i}$. An operator $\mathcal{F}: Y \rightarrow Y$ is called cyclic contraction of implicit relation type if

(I) $Y=\cup_{i=1}^{p} A_{i}$ is a cyclic representation of $Y$ with respect to $\mathcal{F}$;

(II) For any $(x, y) \in A_{i} \times A_{i+1}, i=1,2, \ldots, p\left(\right.$ with $\left.A_{p+1}=A_{1}\right)$,

$$
\begin{aligned}
T(\psi(d(\mathcal{F} x, \mathcal{F} y)), \psi(d(x, y)) & , \psi(d(x, \mathcal{F} x)), \\
& \psi(d(y, \mathcal{F} y)), \psi(d(x, \mathcal{F} y)), \psi(d(y, \mathcal{F} x))) \leq 0
\end{aligned}
$$

where $T \in \digamma$ and $\psi \in \Psi$. 
The following is an example of cyclic contraction of implicit relation type with Example 2.1.

Example 2.6. Let $\mathcal{X}=[0,1]$ with the usual metric. Suppose $\mathcal{A}_{1}=\left[0, \frac{1}{2}\right]$ and $\mathcal{A}_{2}=\left[\frac{1}{2}, 1\right]$ and $\mathcal{X}=\bigcup_{i=1}^{2} \mathcal{A}_{i}$. Define $\mathcal{F}: \mathcal{X} \rightarrow \mathcal{X}$ such that

$$
\mathcal{F} x=\left\{\begin{array}{l}
\frac{1}{2}, x \in[0,1), \\
0, x=1
\end{array}\right.
$$

Clearly, $\mathcal{A}_{1}$ and $\mathcal{A}_{2}$ are closed subsets of $\mathcal{X}$. Moreover $\mathcal{F}\left(\mathcal{A}_{i}\right) \subset \mathcal{A}_{i+1}$ for $i=1,2$, so that $\bigcup_{i=1}^{2} \mathcal{A}_{i}$ is a cyclic representation of $\mathcal{X}$ with respect to $\mathcal{F}$. Furthermore, if $T: \mathbb{R}^{+^{6}} \rightarrow \mathbb{R}^{+}$denote

$$
T\left(t_{1}, t_{2}, t_{3}, t_{4}, t_{5}, t_{6}\right)=t_{1}-c t_{2}-a\left(t_{3}+t_{4}\right)-b \sqrt{t_{5} t_{6}},
$$

where $t_{1}=d(\mathcal{F} x, \mathcal{F} y), t_{2}=d(x, y), t_{3}=d(x, \mathcal{F} x), t_{4}=d(y, \mathcal{F} y), t_{5}=$ $d(x, \mathcal{F} y)$, and $t_{6}=d(y, \mathcal{F} x)$, for all $x, y \in \mathcal{X}$. Also suppose $\psi(t)=\frac{t}{2}$ for $t \geq 0$.

Let $a=\frac{1}{6}, b=\frac{1}{3}$ and $c=\frac{1}{2}$, then $a, b, c$ clearly fulfilled all conditions with $c+\max \{2 a, b\}<1$. Then $T \in \digamma$.

Next we show that the mapping $\mathcal{F}$ is a cyclic contraction of implicit relation type. We shall distinguish the following cases:

For $x \in \mathcal{A}_{1}, y \in \mathcal{A}_{2}\left(\right.$ or $\left.x \in \mathcal{A}_{2}, y \in \mathcal{A}_{1}\right)$.

- When $x \in\left[0, \frac{1}{2}\right]$ and $y \in\left[\frac{1}{2}, 1\right)$, we deduce $d(\mathcal{F} x, \mathcal{F} y)=0$ and equation (4) is trivially satisfied.

- When $x \in\left[0, \frac{1}{2}\right]$ and $y=1$, we deduce $d(\mathcal{F} x, \mathcal{F} y)=\frac{1}{2}$ and

$$
t_{2}=|x-1|, t_{3}=\left|x-\frac{1}{2}\right|, t_{4}=1, t_{5}=x, t_{6}=\frac{1}{2},
$$

then clearly equation (4) holds. Hence the mapping $\mathcal{F}$ is a cyclic contraction of implicit relation type.

Our main result is the following:

Theorem 2.1. Let $(X, d)$ be a complete metric space, $p \in \mathbb{N}, A_{1}, A_{2}, \ldots, A_{p}$ nonempty closed subsets of $X$ and $Y=\cup_{i=1}^{p} A_{i}$. Suppose $\mathcal{F}: Y \rightarrow Y$ is a cyclic contraction of implicit relation type mapping, for some $T \in \digamma$ and $\psi \in \Psi$. Then $\mathcal{F}$ has a unique fixed point. Moreover, the fixed point of $\mathcal{F}$ belongs to $\cap_{i=1}^{p} A_{i}$.

Proof. Let $x_{0} \in A_{1}$ (such a point exists since $A_{1} \neq \emptyset$ ). Define the sequence $\left\{x_{n}\right\}$ in $X$ by:

$$
x_{n+1}=\mathcal{F} x_{n}, \quad n=0,1,2, \ldots
$$

We shall prove that

$$
\lim _{n \rightarrow \infty} d\left(x_{n}, x_{n+1}\right)=0 .
$$


If for some $k$, we have $x_{k+1}=x_{k}$, then (5) follows immediately. So, we can suppose that $d\left(x_{n}, x_{n+1}\right)>0$ for all $n$. From the condition $(I)$, we observe that for all $n$, there exists $i=i(n) \in\{1,2, \ldots, p\}$ such that $\left(x_{n}, x_{n+1}\right) \in$ $A_{i} \times A_{i+1}$. Then, from the condition (II), we have

$$
\begin{aligned}
& T\left(\psi\left(d\left(\mathcal{F} x_{n}, \mathcal{F} x_{n-1}\right)\right), \psi\left(d\left(x_{n}, x_{n-1}\right)\right),\right. \\
& \psi\left(d\left(x_{n}, \mathcal{F} x_{n}\right)\right), \psi\left(d\left(x_{n-1}, \mathcal{F} x_{n-1}\right)\right), \\
& \left.\psi\left(d\left(x_{n}, \mathcal{F} x_{n-1}\right)\right), \psi\left(d\left(x_{n-1}, \mathcal{F} x_{n}\right)\right)\right) \leq 0 .
\end{aligned}
$$

Now since $T \in \digamma$ and we have

$$
T(u, v, v, u, w, 0) \leq 0
$$

for $u=\psi\left(d\left(x_{n+1}, x_{n}\right)\right), v=\psi\left(d\left(x_{n}, x_{n-1}\right)\right), w=\psi\left(d\left(x_{n-1}, x_{n+1}\right)\right)$, it follows from $\left(T_{1}\right)$ that there exists $h \in[0,1)$ such that

$$
\psi\left(d\left(x_{n+1}, x_{n}\right)\right) \leq h \psi\left(d\left(x_{n}, x_{n-1}\right)\right) .
$$

If we continue this procedure, we can have

$$
\psi\left(d\left(x_{n+1}, x_{n}\right)\right) \leq h^{n} \psi\left(d\left(x_{1}, x_{0}\right)\right) .
$$

The sequence $a_{n}:=\psi\left(d\left(x_{n}, x_{n+1}\right)\right), n \geq 1$, of non-negative real numbers, converges to zero. Then there exists a bijection $\sigma: N^{*} \rightarrow N$ such that the sequence $\left\{a_{\sigma(n)}\right\}$ is non-increasing. Since $\psi$ is increasing, it follows that the sequence $\left\{d\left(x_{\sigma(n)}, x_{\sigma(n)+1}\right)\right\}$ of non-negative real numbers is nonincreasing, hence it is convergent. Using the continuity of $\psi$ we obtain $0=$ $\lim _{n \rightarrow \infty} \psi\left(d\left(x_{\sigma(n)}, x_{\sigma(n)+1)}\right)=\psi\left(\lim _{n \rightarrow \infty} d\left(x_{\sigma(n)}, x_{\sigma(n)+1)}\right)\right)\right.$. Since $\psi(t)=0$ if and only if $t=0$, it follows that $\lim _{n \rightarrow \infty} d\left(x_{\sigma(n)}, x_{\sigma(n)+1)}\right)=0$, therefore

$$
\lim _{n \rightarrow \infty} d\left(x_{n+1}, x_{n}\right)=0 .
$$

Next we show that $\left\{x_{n}\right\}$ is a Cauchy sequence. Suppose it is not true. Then we can find a $\delta>0$ and two sequence of integers $\{m(k)\},\{n(k)\}, m(k)>$ $n(k) \geq k$ with

$$
r_{k}=\psi\left(d\left(x_{n(k)}, x_{m(k)}\right)\right) \geq \delta \text { for } k \in\{1,2, \ldots\} .
$$

We may also assume

$$
\psi\left(d\left(x_{m(k)-1}, x_{n(k)}\right)\right)<\delta
$$

by choosing $m(k)$ to be the smallest number exceeding $n(k)$ for which $(7)$ holds. Moreover, since $\psi$ is subadditive and nondecreasing and using (6), (7) and (8) imply

$$
\begin{aligned}
\delta & \leq r_{k}=\psi\left(d\left(x_{m(k)-1}, x_{n(k)}\right)\right) \\
& \leq \psi\left(d\left(x_{m(k)}, x_{m(k)-1}\right)\right)+\psi\left(d\left(x_{m(k)-1}, x_{n(k)}\right)\right) \\
& \leq h^{m(k)-1} \psi\left(d\left(x_{0}, x_{1}\right)\right)+\delta
\end{aligned}
$$


and so

$$
\lim _{k \rightarrow \infty} r_{k}=\delta
$$

Also $\psi$ is subadditive and nondecreasing, we conclude

$$
\begin{aligned}
\delta & \leq \psi\left(d\left(x_{n(k)}, x_{n(k)+1}\right)+d\left(x_{m(k)}, x_{m(k)+1}\right)+d\left(x_{n(k)+1}, x_{m(k)+1}\right)\right) \\
& \left.\leq \psi\left(d\left(x_{n(k)}, x_{n(k)+1}\right)\right)+\psi\left(d\left(x_{m(k)}, x_{m(k)+1}\right)\right)+\psi\left(d\left(x_{n(k)+1}, x_{m(k)+1}\right)\right)\right)
\end{aligned}
$$

we have from (6) that

$$
\delta \leq r_{k}=h^{n(k)} \psi\left(d\left(x_{0}, x_{1}\right)\right)+h^{m(k)} \psi\left(d\left(x_{0}, x_{1}\right)\right)+\psi\left(d\left(x_{m(k)+1}, x_{n(k)+1}\right)\right) .
$$

Using the condition (2) for $x=x_{m(k)}$ and $y=x_{n(k)}$, we have

$$
\begin{aligned}
& T\left(\psi\left(d\left(\mathcal{F} x_{m(k)}, \mathcal{F} x_{n(k)}\right)\right),\right. \psi\left(d\left(x_{m(k)}, x_{n(k)}\right)\right), \\
& \psi\left(d\left(x_{m(k)}, \mathcal{F} x_{m(k)}\right)\right), \psi\left(d\left(x_{n(k)}, \mathcal{F} x_{n(k)}\right)\right), \\
&\left.\psi\left(d\left(x_{m(k)}, \mathcal{F} x_{n(k)}\right)\right), \psi\left(d\left(x_{n(k)}, \mathcal{F} x_{m(k)}\right)\right)\right) \leq 0
\end{aligned}
$$

and so

$$
\begin{array}{r}
T\left(\psi\left(d\left(x_{m(k)+1}, x_{n(k)+1}\right)\right), r_{k}, h^{m(k)}\left(\psi\left(d\left(x_{0}, x_{1}\right)\right)\right), h^{n(k)}\left(\psi\left(d\left(x_{0}, x_{1}\right)\right)\right),\right. \\
\left.r_{k}+h^{n(k)}\left(\psi\left(d\left(x_{0}, x_{1}\right)\right)\right), r_{k}+h^{m(k)}\left(\psi\left(d\left(x_{0}, x_{1}\right)\right)\right)\right) \leq 0 .
\end{array}
$$

Now passing to the limit as $k \rightarrow \infty$ in above and using (9) we have, by continuity of $T$ and $\Psi$ that

$$
T\left(\lim _{k \rightarrow \infty} \psi\left(d\left(x_{m(k)+1}, x_{n(k)+1}\right)\right), \delta, 0,0, \delta, \delta\right) \leq 0 .
$$

From $\left(T_{1}\right)$, we have $\lim _{k \rightarrow \infty} \psi\left(d\left(x_{m(k)+1}, x_{n(k)+1}\right)\right) \leq h(\psi(\delta))$. Therefore from (10) we have $\delta \leq h(\psi(\delta))$. This is a contradiction since $h \in[0,1)$ and $\psi(t)<t$ for $t>0$. Thus $\left\{x_{n}\right\}$ is a Cauchy sequence in $\mathcal{X}$.

Since $(X, d)$ is complete, there exists $x^{*} \in X$ such that

$$
\lim _{n \rightarrow \infty} x_{n}=x^{*} .
$$

We shall prove that

$$
x^{*} \in \bigcap_{i=1}^{p} A_{i} .
$$

From condition $(I)$, and since $x_{0} \in A_{1}$, we have $\left\{x_{n p}\right\}_{n \geq 0} \subseteq A_{1}$. Since $A_{1}$ is closed, from (11), we get that $x^{*} \in A_{1}$. Again, from the condition $(I)$, we have $\left\{x_{n p+1}\right\}_{n \geq 0} \subseteq A_{2}$. Since $A_{2}$ is closed, from (11), we get that $x^{*} \in A_{2}$. Continuing this process, we obtain (12). 
Now, we shall prove that $x^{*}$ is a fixed point of $\mathcal{F}$. Indeed, from (12), since for all $n$, there exists $i(n) \in\{1,2, \ldots, p\}$ such that $x_{n} \in A_{i(n)}$, applying $(I I)$ with $x=x^{*}$ and $y=x_{n}$, we obtain

$$
\begin{aligned}
T\left(\psi\left(d\left(\mathcal{F} x^{*}, \mathcal{F} x_{n}\right)\right), \psi\right. & \left(d\left(x^{*}, x_{n}\right)\right), \psi\left(d\left(x^{*}, \mathcal{F} x^{*}\right)\right), \\
& \left.\psi\left(d\left(x_{n}, \mathcal{F} x_{n}\right)\right), \psi\left(d\left(x^{*}, \mathcal{F} x_{n}\right)\right), \psi\left(d\left(x_{n}, \mathcal{F} x^{*}\right)\right)\right) \leq 0
\end{aligned}
$$

so letting $n \rightarrow \infty$ from the last inequality with the property of $\psi$ we have

$$
T\left(\psi\left(d\left(\mathcal{F} x^{*}, x^{*}\right)\right), 0, \psi\left(d\left(x^{*}, \mathcal{F} x^{*}\right)\right), 0,0, \psi\left(d\left(x^{*}, \mathcal{F} x^{*}\right)\right)\right) \leq 0 .
$$

By $\left(T_{1}\right)$ it follows that $\psi\left(d\left(x^{*}, \mathcal{F} x^{*}\right)\right) \leq 0$ and so $x^{*}=\mathcal{F} x^{*}$, that is, $x^{*}$ is a fixed point of $\mathcal{F}$.

Finally, we prove that $x^{*}$ is the unique fixed point of $\mathcal{F}$. Assume that $y^{*}$ is another fixed point of $\mathcal{F}$, that is, $\mathcal{F} y^{*}=y^{*}$. By the condition $(I)$, this implies that $y^{*} \in \bigcap_{i=1}^{p} A_{i}$. Then we can apply $(I I)$ for $x=x^{*}$ and $y=y^{*}$. We obtain

$$
\begin{aligned}
T\left(\psi\left(d\left(\mathcal{F} x^{*}, \mathcal{F} y^{*}\right)\right), \psi(\right. & \left.\left(x^{*}, y^{*}\right)\right), \psi\left(d\left(x^{*}, \mathcal{F} x^{*}\right)\right), \\
& \left.\psi\left(d\left(y^{*}, \mathcal{F} y^{*}\right)\right), \psi\left(d\left(x^{*}, \mathcal{F} y^{*}\right)\right), \psi\left(d\left(y^{*}, \mathcal{F} x^{*}\right)\right)\right) \leq 0 .
\end{aligned}
$$

Since $x^{*}$ and $y^{*}$ are fixed points of $\mathcal{F}$, we can show easily that $x^{*} \neq y^{*}$. If $d\left(x^{*}, y^{*}\right)>0$, we get

$$
T\left(\psi\left(d\left(x^{*}, y^{*}\right)\right), \psi\left(d\left(x^{*}, y^{*}\right)\right), 0,0, \psi\left(d\left(x^{*}, y^{*}\right)\right), \psi\left(d\left(y^{*}, x^{*}\right)\right)\right) \leq 0 .
$$

a contradiction of $\left(T_{2}\right), \psi\left(d\left(x^{*}, y^{*}\right)\right)=0$; hence $d\left(x^{*}, y^{*}\right)=0$, that is, $x^{*}=$ $y^{*}$. Thus we proved the uniqueness of the fixed point.

Remark 2.1. Note that if we take

$T_{3}$ : there exists $h \in[0,1)$ such that for all $u, v, w \geq 0$

$$
T(u, v, v, u, w, 0) \leq 0
$$

or

$$
T(u, v, u, v, 0, w) \leq 0
$$

implies $u \leq h v$, instead of $T_{1}$ in Theorem 2.1, again we can have the same result.

In the following, we deduce some fixed point theorems from our main result given by Theorem 2.1 .

If we take $p=1$ and $A_{1}=X$ in Theorem 2.1 , then we get immediately the following fixed point theorem for one map due to Popa and Mocanu [15, Theorem 1]: 
Corollary 2.1. Let $(X, d)$ be a complete metric space and let $\mathcal{F}: X \rightarrow X$ satisfy the following condition: there exists $T \in \digamma$ and $\psi \in \Psi$ such that

$$
\begin{aligned}
T(\psi(d(\mathcal{F} x, \mathcal{F} y)), \psi(d(x, y)), & \psi(d(x, \mathcal{F} x)), \\
& \psi(d(y, \mathcal{F} y)), \psi(d(x, \mathcal{F} y)), \psi(d(y, \mathcal{F} x))) \leq 0,
\end{aligned}
$$

for all $x, y \in X$. Then $\mathcal{F}$ has a unique fixed point.

If $\psi$ is the identity in Corollary 2.1, we have following:

Corollary 2.2. Let $(X, d)$ be a complete metric space and let $\mathcal{F}: X \rightarrow X$ satisfy the following condition: there exists $T \in \digamma$ such that

$$
T(d(\mathcal{F} x, \mathcal{F} y), d(x, y), d(x, \mathcal{F} x), d(y, \mathcal{F} y), d(x, \mathcal{F} y), d(y, \mathcal{F} x)) \leq 0,
$$

for all $x, y \in X$. Then $\mathcal{F}$ has a unique fixed point.

If we combine Theorem 2.1 with Example 2.1, we obtain the following result.

Corollary 2.3. Let $(X, d)$ be a complete metric space, $p \in \mathbb{N}, A_{1}, A_{2}, \ldots, A_{p}$ nonempty closed subsets of $X, Y=\cup_{i=1}^{p} A_{i}$ and $\mathcal{F}: Y \rightarrow Y$. Suppose

$(I)^{\prime} Y=\cup_{i=1}^{p} A_{i}$ is a cyclic representation of $Y$ with respect to $\mathcal{F}$;

$(I I)^{\prime}$ For any $(x, y) \in A_{i} \times A_{i+1}, i=1,2, \ldots, p\left(\right.$ with $\left.A_{p+1}=A_{1}\right)$,

$$
d(\mathcal{F} x, \mathcal{F} y) \leq c d(x, y)+a(d(x, \mathcal{F} x)+d(y, \mathcal{F} y))+b \sqrt{d(x, \mathcal{F} y) . d(y, \mathcal{F} x)}
$$

where $c>0, a, b \geq 0$ and $c+\max \{2 a, b\}<1$. Then $\mathcal{F}$ has a unique fixed point. Moreover, the fixed point of $\mathcal{F}$ belongs to $\cap_{i=1}^{p} A_{i}$.

If we combine Theorem 2.1 with Example 2.3, we obtain the following result.

Corollary 2.4. Let $(X, d)$ be a complete metric space, $p \in \mathbb{N}, A_{1}, A_{2}, \ldots, A_{p}$ nonempty closed subsets of $X, Y=\cup_{i=1}^{p} A_{i}$ and $\mathcal{F}: Y \rightarrow Y$. Suppose

$(I)^{\prime} Y=\cup_{i=1}^{p} A_{i}$ is a cyclic representation of $Y$ with respect to $\mathcal{F}$;

$(I I)^{\prime}$ For any $(x, y) \in A_{i} \times A_{i+1}, i=1,2, \ldots, p\left(\right.$ with $\left.A_{p+1}=A_{1}\right)$,

$$
\begin{aligned}
\psi(d(\mathcal{F} x, \mathcal{F} y)) \leq & a \psi(d(x, y))+b \psi(d(x, \mathcal{F} x)) \\
& +c \psi(d(y, \mathcal{F} y))+d \min \{\psi(d(x, \mathcal{F} y)), \psi(d(y, \mathcal{F} x))\}
\end{aligned}
$$

where $a>0, b, c, d \geq 0$ and $a+\max \{b+c, d\}<1$, and $\psi \in \Psi$. Then $\mathcal{F}$ has a unique fixed point. Moreover, the fixed point of $\mathcal{F}$ belongs to $\cap_{i=1}^{p} A_{i}$.

Remark 2.2. Corollary 2.4 is an extension to Theorem 2.1 in $[4,3,22,23]$.

If the altering distance $\psi$ be the identity in Theorem 2.1, we have following: 
Corollary 2.5. Let $(X, d)$ be a complete metric space, $p \in \mathbb{N}, A_{1}, A_{2}, \ldots, A_{p}$ nonempty closed subsets of $X, Y=\cup_{i=1}^{p} A_{i}$ and $\mathcal{F}: Y \rightarrow Y$. Suppose that there exists $T \in \digamma$ such that

$(I)^{\prime} Y=\cup_{i=1}^{p} A_{i}$ is a cyclic representation of $Y$ with respect to $\mathcal{F}$;

$(I I)^{\prime}$ For any $(x, y) \in A_{i} \times A_{i+1}, i=1,2, \ldots, p\left(\right.$ with $\left.A_{p+1}=A_{1}\right)$,

$$
T(d(\mathcal{F} x, \mathcal{F} y), d(x, y), d(x, \mathcal{F} x), d(y, \mathcal{F} y), d(x, \mathcal{F} y), d(y, \mathcal{F} x)) \leq 0 .
$$

Then $\mathcal{F}$ has a unique fixed point. Moreover, the fixed point of $\mathcal{F}$ belongs to $\cap_{i=1}^{p} A_{i}$.

The following example demonstrates the validity of Corollary 2.4 obtained by modifying the example from [6].

Example 2.7. Let $\mathcal{X}=\mathbb{R}$ with the usual metric. Suppose $\mathcal{A}_{1}=[-1,0]=$ $\mathcal{A}_{3}$ and $\mathcal{A}_{2}=[0,1]=\mathcal{A}_{4}$ and $\mathcal{Y}=\bigcup_{i=1}^{4} \mathcal{A}_{i}$. Define $\mathcal{F}: \mathcal{Y} \rightarrow \mathcal{Y}$ by $\mathcal{F} x=\frac{-x}{6}$ for all $x \in \mathcal{Y}$. Clearly, $\mathcal{A}_{i}(i=1,2,3,4)$ are closed subsets of $\mathcal{X}$. Moreover $\mathcal{F}\left(\mathcal{A}_{i}\right) \subset \mathcal{A}_{i+1}$ for $i=1,2,3,4$ so that $\bigcup_{i=1}^{4} \mathcal{A}_{i}$ is a cyclic representation of $\mathcal{Y}$ with respect to $\mathcal{F}$. Moreover, mapping $\mathcal{F}$ is a implicit relation type cyclic contractive, where $T: \mathbb{R}^{+^{6}} \rightarrow \mathbb{R}^{+}$defined by

$$
T\left(t_{1}, t_{2}, t_{3}, t_{4}, t_{5}, t_{6}\right)=t_{1}-a t_{2}-b t_{3}-c t_{4}-d \min \left\{t_{5}, t_{6}\right\},
$$

where $t_{1}=d(\mathcal{F} x, \mathcal{F} y), t_{2}=d(x, y), t_{3}=d(x, \mathcal{F} x), t_{4}=d(y, \mathcal{F} y), t_{5}=$ $d(x, \mathcal{F} y)$, and $t_{6}=d(y, \mathcal{F} x)$, for all $x, y \in \mathcal{X}$. Then $T \in \mathcal{F}$. Indeed, to see this fact we examine following cases:

Equation (17) reduces to

$$
\begin{aligned}
& \psi(d(\mathcal{F} x, \mathcal{F} y))=\psi\left(\frac{|x-y|}{6}\right) \\
\leq & a \psi(d(x, y))+b \psi\left(\frac{5|x|}{6}\right)+c \psi\left(\frac{5|y|}{6}\right)+d \min \left\{\psi\left(\left|x+\frac{y}{6}\right|\right), \psi\left(\left|y+\frac{x}{6}\right|\right)\right\} .
\end{aligned}
$$

Let $a=\frac{1}{2}, b=c=d=\frac{1}{6}$, then $a, b, c, d$ clearly fulfilled all conditions with $a+\max \{b+c, d\}<1$. Let $\psi(t)=\frac{t}{2}$ for $t \geq 0$.

- For $x \in \mathcal{A}_{1}, y \in \mathcal{A}_{2}$.

(i) Suppose $x=-1$ and $y=0$. Then equation (16) holds as it reduces to $\frac{1}{6}<\frac{2}{3}$.

(ii) Suppose $x=0$ and $y=1$. Then equation (16) holds as it reduces to $\frac{1}{6}<\frac{2}{3}$.

(iii) Suppose $x=-1$ and $y=1$. Then equation (16) holds as it reduces to $\frac{1}{3}<\frac{51}{36}$.

- For $x \in \mathcal{A}_{2}, y \in \mathcal{A}_{1}$. 
(i) Suppose $x=\frac{1}{2}$ and $y=\frac{-1}{2}$. Then equation (16) holds as it reduces to $\frac{1}{6}<\frac{51}{72}$.

(ii) Suppose $x=1$ and $y=-1$. Then equation (16) holds as it reduces to $\frac{1}{3}<\frac{51}{36}$.

- For $x=y, d(\mathcal{F} x, \mathcal{F} y)=0$. Then equation (16) trivially holds.

Similarly other cases can be verified. Therefore, all conditions of Corollary 2.4 are satisfied, and so $\mathcal{F}$ has a fixed point (which is $z=0 \in \bigcap_{i=1}^{4} \mathcal{A}_{i}$ ).

We illustrate Corollary 2.3 by an example which is obtained by modifying the one from [10].

Example 2.8. Let $\mathcal{X}=\mathbb{R}^{+^{3}}$ and we define $d: \mathcal{X} \times \mathcal{X} \rightarrow[0,1)$ by

$$
d(x, y)=\left|x_{1}-y_{1}\right|+\left|x_{2}-y_{2}\right|+\left|x_{3}-y_{3}\right|,
$$

for $x=\left(x_{1}, x_{2}, x_{3}\right), y=\left(y_{1}, y_{2}, y_{3}\right) \in \mathcal{X}$, and let $\mathcal{A}=\left\{(x, 0,0): x \in \mathbb{R}^{+}\right\}$, $\mathcal{B}=\left\{(0, y, 0): y \in \mathbb{R}^{+}\right\}, \mathcal{C}=\left\{(0,0, z): z \in \mathbb{R}^{+}\right\}$, be three subsets of $\mathcal{X}$.

Define $\mathcal{F}: \mathcal{A} \cup \mathcal{B} \cup \mathcal{C} \rightarrow \mathcal{A} \cup \mathcal{B} \cup \mathcal{C}$ by

$$
\begin{aligned}
& \mathcal{F}((x, 0,0))=\left(0, \frac{1}{6} x, 0\right) ; \text { for all } x \in \mathbb{R}^{+} ; \\
& \mathcal{F}((0, y, 0))=\left(0,0, \frac{1}{6} y\right) ; \text { for all } y \in \mathbb{R}^{+} ; \\
& \mathcal{F}((0,0, z))=\left(\frac{1}{6} z, 0,0\right) ; \text { for all } x \in \mathbb{R}^{+} ;
\end{aligned}
$$

Let the function $T: \mathbb{R}^{+^{6}} \rightarrow \mathbb{R}^{+}$be defined by

$$
T\left(t_{1}, t_{2}, t_{3}, t_{4}, t_{5}, t_{6}\right)=t_{1}-c t_{2}-a\left(t_{3}+t_{4}\right)-b \sqrt{t_{5} t_{6}},
$$

where $t_{1}=d(\mathcal{F} x, \mathcal{F} y), t_{2}=d(x, y), t_{3}=d(x, \mathcal{F} x), t_{4}=d(y, \mathcal{F} y), t_{5}=$ $d(x, \mathcal{F} y)$, and $t_{6}=d(y, \mathcal{F} x)$, for all $x, y \in \mathcal{X}$.

Let $a=\frac{1}{6}, b=\frac{1}{3}$ and $c=\frac{1}{2}$, then $a, b, c$ clearly fulfilled all conditions with $c+\max \{2 a, b\}<1$. Then $T \in \digamma$.

By routine calculation, one can prove that $\mathcal{F}$ is a cyclic contraction satisfying implicit relation type mapping for $\psi(t)=\frac{1}{3} t$ for $t \geq 0$. Therefore, all conditions of Corollary 2.3 are satisfied, and so $\mathcal{F}$ has a fixed point (which is $(0,0,0) \in \bigcap_{i=1}^{3} \mathcal{A} \cup \mathcal{B} \cup \mathcal{C}$.)

\section{An application to integral EQUATions}

In this section, we apply Corollary 2.4 to study the existence and uniqueness of solutions to a class of nonlinear integral equations.

We consider the nonlinear integral equation

$$
u(t)=\int_{0}^{\mathbf{T}} G(t, s) f(s, u(s)) d s, \quad \text { for all } t \in[0, \mathbf{T}],
$$


where $\mathbf{T}>0, f:[0, \mathbf{T}] \times \mathbb{R} \rightarrow \mathbb{R}$ and $G:[0, \mathbf{T}] \times[0, \mathbf{T}] \rightarrow[0, \infty)$ are continuous functions.

Let $\mathcal{X}=C([0, \mathbf{T}])$ be the set of real continuous functions on $[0, T]$. We endow $\mathcal{X}$ with the standard metric

$$
d_{\infty}(u, v)=\max _{t \in[0, \mathbf{T}]}|u(t)-v(t)|, \text { for all } u, v \in \mathcal{X} .
$$

It is well known that $\left(\mathcal{X}, d_{\infty}\right)$ is a complete metric space.

Let $(\alpha, \beta) \in \mathcal{X}^{2},\left(\alpha_{0}, \beta_{0}\right) \in \mathbb{R}^{2}$ such that

$$
\alpha_{0} \leq \alpha \leq \beta \leq \beta_{0}
$$

We suppose that for all $t \in[0, \mathbf{T}]$, we have

$$
\alpha(t) \leq \int_{0}^{\mathbf{T}} G(t, s) f(s, \beta(s)) d s
$$

and

$$
\beta(t) \geq \int_{0}^{\mathbf{T}} G(t, s) f(s, \alpha(s)) d s .
$$

We suppose that for all $s \in[0, \mathbf{T}], f(s, \cdot)$ is a decreasing function, that is,

$$
x, y \in \mathbb{R}, x \geq y \Longrightarrow f(s, x) \leq f(s, y) .
$$

We suppose that

$$
\sup _{t \in[0, \mathbf{T}]} \int_{0}^{\mathbf{T}} G(t, s) d s \leq 1 .
$$

Finally, we suppose that for all $s \in[0,1]$, for all $x, y \in \mathbb{R}$ with $x \leq \beta_{0}$ and $y \geq \alpha_{0}$ or $x \geq \alpha_{0}$ and $y \leq \beta_{0}$,

$$
\begin{aligned}
& |f(s, x)-f(s, y)| \\
& \quad \leq a d(x, y)+b d(x, \mathcal{F} x)+c d(y, \mathcal{F} y)+d \min \{d(x, \mathcal{F} y), d(y, \mathcal{F} x)\},
\end{aligned}
$$

where $a>0, b, c, d \geq 0$ and $a+\max \{b+c, d\}<1$.

Now, define the set

$$
\mathcal{C}=\{u \in C([0, \mathbf{T}]): \alpha \leq u \leq \beta\} .
$$

We have the following result.

Theorem 3.1. Under the assumptions (19)-(24), problem (18) has one and only one solution $u^{*} \in \mathcal{C}$.

Proof. Define the closed subsets of $\mathcal{X}, \mathcal{A}_{1}$ and $\mathcal{A}_{2}$ by

$$
\mathcal{A}_{1}=\{u \in \mathcal{X}: u \leq \beta\}
$$

and

$$
\mathcal{A}_{2}=\{u \in \mathcal{X}: u \geq \alpha\}
$$


Define the mapping $\mathcal{F}: \mathcal{X} \rightarrow \mathcal{X}$ by

$$
\mathcal{F} u(t)=\int_{0}^{\mathbf{T}} G(t, s) f(s, u(s)) d s, \text { for all } t \in[0, \mathbf{T}] .
$$

We shall prove that

$$
\mathcal{F}\left(\mathcal{A}_{1}\right) \subseteq \mathcal{A}_{2} \text { and } \mathcal{F}\left(A_{2}\right) \subseteq A_{1}
$$

Let $u \in \mathcal{A}_{1}$, that is,

$$
u(s) \leq \beta(s), \text { for all } s \in[0, \mathbf{T}] .
$$

Using condition (22), since $G(t, s) \geq 0$ for all $t, s \in[0, \mathbf{T}]$, we obtain that

$$
G(t, s) f(s, u(s)) \geq G(t, s) f(s, \beta(s)) \text {, for all } t, s \in[0, \mathbf{T}] .
$$

The above inequality with condition (20) implies that

$$
\int_{0}^{\mathbf{T}} G(t, s) f(s, u(s)) d s \geq \int_{0}^{\mathbf{T}} G(t, s) f(s, \beta(s)) d s \geq \alpha(t),
$$

for all $t \in[0, \mathbf{T}]$. Then we have $\mathcal{F} u \in \mathcal{A}_{2}$.

Similarly, let $u \in \mathcal{A}_{2}$, that is,

$$
u(s) \geq \alpha(s), \text { for all } s \in[0, \mathbf{T}] .
$$

Using condition (22), since $G(t, s) \geq 0$ for all $t, s \in[0, \mathbf{T}]$, we obtain that

$$
G(t, s) f(s, u(s)) \leq G(t, s) f(s, \alpha(s)) \text {, for all } t, s \in[0, \mathbf{T}] .
$$

The above inequality with condition (21) implies that

$$
\int_{0}^{\mathbf{T}} G(t, s) f(s, u(s)) d s \leq \int_{0}^{\mathbf{T}} G(t, s) f(s, \alpha(s)) d s \leq \beta(t),
$$

for all $t \in[0, \mathbf{T}]$. Then we have $\mathcal{F} u \in \mathcal{A}_{1}$. Finally, we deduce that (25) holds.

Now, let $(u, v) \in \mathcal{A}_{1} \times \mathcal{A}_{2}$, that is, for all $t \in[0, \mathbf{T}]$,

$$
u(t) \leq \beta(t), \quad v(t) \geq \alpha(t) .
$$

This implies from condition (19) that for all $t \in[0, \mathbf{T}]$,

$$
u(t) \leq \beta_{0}, \quad v(t) \geq \alpha_{0} .
$$


Now, using conditions (23) and (24), we can write that for all $t \in[0, \mathbf{T}]$, we have

$$
\begin{aligned}
& |\mathcal{F} u-\mathcal{F} v|(t) \leq \int_{0}^{\mathbf{T}} G(t, s)|f(s, u(s))-f(s, v(s))| d s \\
& \leq \int_{0}^{\mathbf{T}} G(t, s)[a|u(s)-v(s)|+b|u(s)-\mathcal{F} u(s)|+c|v(s)-\mathcal{F} v(s)| \\
& +d \min \{|u(s)-\mathcal{F} v(s)|,|v(s)-\mathcal{F} u(s)|\}] d s \\
& \leq\left[a d_{\infty}(u, v)+b d_{\infty}(u, \mathcal{F} u)+c d_{\infty}(v, \mathcal{F} v)\right. \\
& \left.+d \min \left\{d_{\infty}(u, \mathcal{F} v), d_{\infty}(v, \mathcal{F} u)\right\}\right] \int_{0}^{\mathbf{T}} G(t, s) d s \\
& \leq a d_{\infty}(u, v)+b d_{\infty}(u, \mathcal{F} u)+c d_{\infty}(v, \mathcal{F} v)+d \min \left\{d_{\infty}(u, \mathcal{F} v), d_{\infty}(v, \mathcal{F} u)\right\} .
\end{aligned}
$$

This implies that

$$
\begin{aligned}
\psi\left(d_{\infty}(\mathcal{F} u, \mathcal{F} v)\right) \leq a \psi\left(d_{\infty}(u, v)\right) & +b \psi\left(d_{\infty}(u, \mathcal{F} u)\right)+c \psi\left(d_{\infty}(v, \mathcal{F} v)\right) \\
& +d \min \left\{\psi\left(d_{\infty}(u, \mathcal{F} v)\right), \psi\left(d_{\infty}(v, \mathcal{F} u)\right)\right\},
\end{aligned}
$$

where $\psi(t)=t$. Using the same technique, we can show that the above inequality holds also if we take $(u, v) \in \mathcal{A}_{2} \times \mathcal{A}_{1}$.

Now, all conditions of Corollary 2.4 are satisfied (with $p=2$ ), and we deduce that $\mathcal{F}$ has a unique fixed point $u^{*} \in \mathcal{A}_{1} \cap \mathcal{A}_{2}=\mathcal{C}$, that is, $u^{*} \in \mathcal{C}$ is the unique solution to (18).

\section{REFERENCES}

[1] S. Banach, Sur les opérations dans les ensembles abstraits et leur application aux equations integrales, Fund. Math., 3 (1922) 133-181.

[2] H. K. Nashine, New fixed point theorems for mappings satisfying generalized weakly contractive condition with weaker control functions, Ann. Polonici Math., 104 (2012), 109-119.

[3] W. A. Kirk, P. S. Srinivasan and P. Veeramani, Fixed points for mappings satisfying cyclical contractive conditions, Fixed Point Theory, 4 (1) (2003), 79-89.

[4] M. Pacurar and I. A. Rus, Fixed point theory for cyclic $\varphi$-contractions, Nonlinear Anal., 72 (2010), 1181-1187.

[5] R. P. Agarwal, M. A. Alghamd and, N. Shahzad, Fixed point theory for cyclic generalized contractions in partial metric spaces, Fixed Point Theory Appl., 2012 (40) (2012), doi:10.1186/1687-1812-2012-40.

[6] E. Karapinar, Fixed point theory for cyclic weak $\phi$-contraction, Appl. Math. Lett., 24 (6) (2011), 822-825.

[7] E. Karapınar and K. Sadaranagni, Fixed point theory for cyclic ( $\phi-\psi)$-contractions, Fixed Point Theory Appl., 2011, 69 (2011), doi:10.1186/1687-1812-2011-69.

[8] M. A. Petric, Some results concerning cyclical contractive mappings, General Math., 18 (4) (2010), 213-226. 
[9] I. A. Rus, Cyclic representations and fixed points, Ann. T. Popoviciu, Seminar Funct. Eq. Approx. Convexity, 3 (2005), 171-178.

[10] C. M. Chen, Fixed point theory for the cyclic weaker Meir-Keeler function in complete metric spaces, Fixed Point Theory Appl., 2012, 2012:17, doi:10.1186/1687-1812-201217.

[11] H. K. Nashine, Z. Kadelburg and P. Kumam, Implicit-relation-type cyclic contractive mappings and applications to integral equations, Abstr. Appl. Anal., Volume 2012, Article ID 386253, 15 pages.

[12] V. Popa, A fixed point theorem for mapping in d-complete topological spaces, Math. Moravica, 3 (1999), 43-48.

[13] I. Altun and D. Turkoglu, Some fixed point theorems for weakly compatible multivalued mappings satisfying an implicit relation, Filomat, 22 (1) (2008), 13-23.

[14] I. Altun and D. Turkoglu, Some fixed point theorems for weakly compatible mappings satisfying an implicit relation, Taiwanese J. Math., 13 (4) (2009), 1291-1304.

[15] I. Altun and H. Simsek, Some fixed point theorems on ordered metric spaces and application, Fixed Point Theory Appl., 2010 (2010), Article ID 621492, 17 pages.

[16] M. Imdad, S. Kumar and M. S. Khan, Remarks on some fixed point theorems satisfying implicit relations, Rad. Math., 11 (1) (2002), 135-143.

[17] V. Popa, A general coincidence theorem for compatible multivalued mappings satisfying an implicit relation, Demontsratio Math., 33 (1) (2000), 159-164.

[18] V. Popa and M. Mocanu, Altering distance and common fixed points under implicit relations, Hacet. J. Math. Stat., 38 (3) (2009), 329-337.

[19] A. Aliouche and A. Djoudi, Common fixed point theorems for mappings satisfying an implicit relation without decreasing assumption, Hacet. J. Math. Stat., 36 (1) (2007), 11-18.

[20] S. Sharma and B. Desphande, On compatible mappings satisfying an implicit relation in common fixed point consideration, Tamkang J. Math., 33 (3) (2002), 245-252.

[21] M. S. Khan, M. Swaleh and S. Sessa, Fixed point theorems by altering distances between the points, Bull. Austral. Math. Soc., 30 (1984), 1-9.

[22] D. W. Boyd and J. S. Wong, On nonlinear contractions, Proc. Amer. Math. Soc., 20 (1969), 458-469.

[23] G. E. Hardy and T. D. Rogers, A Generalization of a fixed point theorem of Reich, Canad. Math. Bull., 16 (2) (1973), 201-206.

(Received: January 14, 2014)

Department of Mathematics

Disha Institute of Management and Technology Satya Vihar, Vidhansabha-Chandrakhuri Marg Mandir Hasaud, Raipur-492101(Chhattisgarh)

India

drhknashine@gmail.com

hemantnashine@gmail.com 